\title{
BlotCycler consistently shows an increase in western blot
}

\section{signal intensity}

Many biological researchers know the capricious nature of western blotting. Although the process may be performed over and over again with the same reagents and protocols, results can differ clouding the objective of the experiment.

A device exists that provides labs with confidence in obtaining consistent results while reducing the time intensive tasks of manually processing blots. The human error evident in blot staining is also reduced or eliminated; a clear advantage for applications in which western blotting is used to confirm findings or discover new facts.

BlotCycler performs all western blot staining steps automatically, from blocking through final washing. The system allows users to program the precise duration of each step as well as the number of washes. Uniquely shaped trays and optimal agitation speeds efficiently mix reagents, while gravity-fed fluidics provide consistent and reproducible dispensing of solutions. Numerous experiments have proven BlotCycler's ability to consistently deliver standardized western blotting results.

Results of western blot experiments performed manually compared to automated procedures using BlotCycler are provided in Figure A at right. Signal intensity is clearly brighter on the blot processed using BlotCycler.

For the comparison shown here, BlotCycler performed all processing steps uninterrupted at $4^{\circ} \mathrm{C}$. For the manual procedure, the primary antibody was incubated overnight at $4^{\circ} \mathrm{C}$ in the cold room, but remaining processing steps were performed at room temperature.

Automated performance allows standardization: The timing and consistency of solution changes, efficient washing, and elimination of operator error permits BlotCycler to deliver highly reproducible results.

Standardized performance allows researchers to determine the optimal concentrations of both primary and secondary antibodies, an essential task to obtaining the most accurate and informative results. BlotCycler will process from 1 to 24 blots, and can handle 6 different primary and secondary antibodies while running up to 2 protocols simultaneously. The inherent variation of manually processing multiple blots is avoided, and researchers can easily generate the titration curves for both primary and secondary antibodies.

New application information is published continuously.

Call toll free, 1-888-490-4443 or visit www.blotcycler.com to view additional applications.

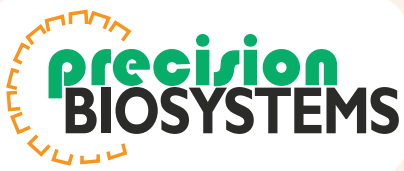

